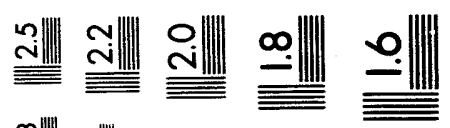

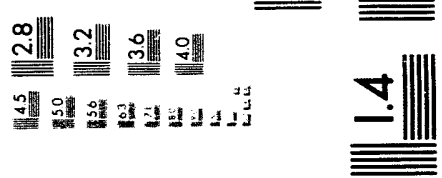

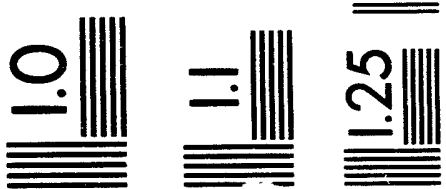



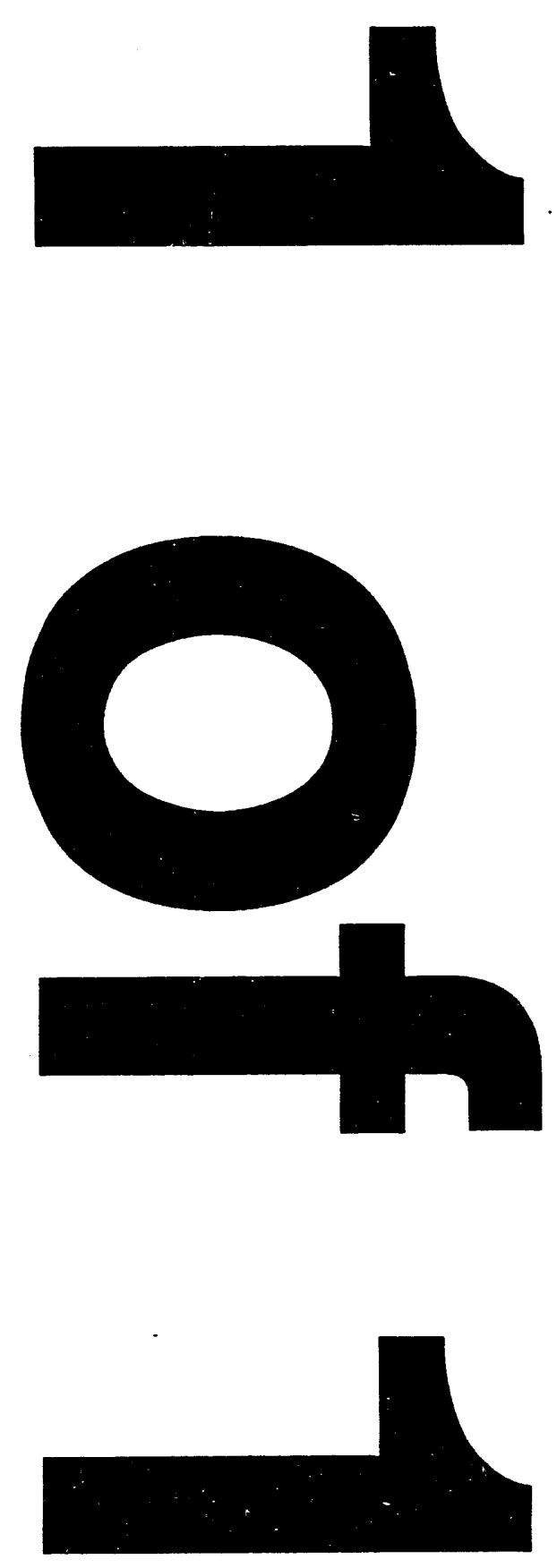
Log Number: 193

Conf-94010/- 7

\title{
TESTING AND RESEARCH CAPABILITIES AT THE SANDIA FAST PULSED REACTOR FACILITY
}

\author{
Donald T. Berry \\ Sandia National Laboratories \\ Department 6521 \\ P.O. Box 5800
}

Albuquerque, New Mexico 87185-5800

(505) $845-3142$

CAMERA READY MANUSCRIPT prepared for:

Eleventh Symposium

on Space Nuclear Power and Propulsion

Albuquerque, New Mexico

9-13 January 1994

initial submission: 30 September 1993

final submission:

author to whom correspondence should be sent: Donald T. Berry
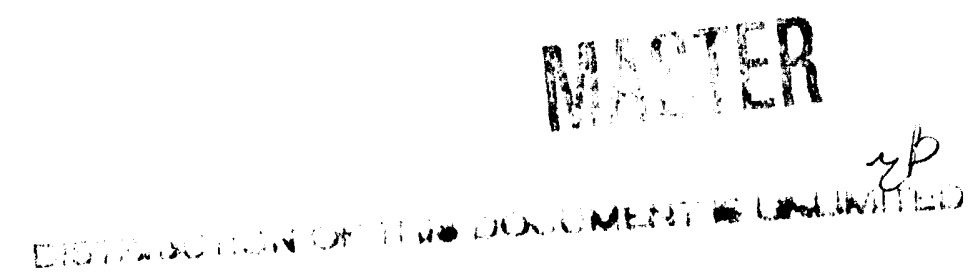


\title{
TESTING AND RESEARCH CAPABILITIES AT THE SANDIA FAST PULSED REACTOR FACILTTY
}

\author{
Donald T. Berry \\ Sandia National Laboratories \\ Department 6521 \\ P.0. Box 5800 \\ Albuquerque, New Mexico 87185-5800
}

(505) 845-3142

\begin{abstract}
A wide variety of space-based system components have been qualified for use through neutron irradiation testing performed at the Sandia Pulsed Reactor (SPR) Facility. The SPR Facility is the operating location for two fast burst reactors, SPR II and SPR III, which have been used to induce neutron and gamma damage in electronic components and other materials for customers in the Department of Energy, Department of Defense, NASA and the private sector. In addition to the pulse mode of operation, during which peak fluxes of up to $1 \mathrm{e} 19 \mathrm{n} / \mathrm{cm}^{2}$-s are achieved, the steady state mode allows for the long terni irradiation of components and systems in a fast neutron environment at a flux of up to $5 \mathrm{e} 11 \mathrm{n} / \mathrm{cm}^{2}$-s. The SPR reactors are operated in a 9.2 meter diameter exposure cell, or Kiva, suitable for the irradiation of large cest articles external to the reactors. Currently, a new upgraded version of SPR III (SPR IIIM) is in fabrication; a unique feature of SPR IIIM is its $19 \mathrm{~cm}$ (usable diameter) central irradiation cavity, the largest of any U.S. fast burst reactor. An improved cooling system permits continuous operation at power levels in excess of $20 \mathrm{~kW}_{t}$. The SPR Facility is also the operating site fo: a critical assembly which was used to characterize prototypic fuels in arrays appropriate for the Space Nuclear Thermal Propulsion Program. Work continues on use of the facility to design, build, and operate critical assemblies for a diverse customer base.
\end{abstract}

\section{INTRODUCTION}

For over thirty years, Sandia National Laboratories (SNL) has operated fast burst reactors for the U.S. Department of Energy (DOE) in support of nuclear weapons development programs. These have included the SPR, SPR II, and SPR III; an upgraded version of SPR III (SPR IIIM) is in fabrication. Though built to service national defense needs, the user community of the reactors has increased in scope, as an assortment of both military and nonmilitary applications have been discovered. The resources of the reactor operating and support staffs have been continually challenged to adapt to new requirements. Accordingly, the Sandia Pulsed Reactor Facility (Figure 1) has diversified into the design, construction, and operation of a critical assembly used to evaluate advanced fuels intended for use in a space nuclear thermal propulsion reactor. Additional benchmark criticals are being planned at the facility. The SPR III reactor has been characterized as a benchmark reference source for dosimetry and electronics testing.

\section{SPR/SPR II}

The original SPR was designed and fabricated by Los Alamos National Laboratories (LANL) and operated at SNL between 1960 and 1967. As test requirements were adjusted to coincide with more severe weapon threat scenarios, it became clear that a reactor with improved capabilities was needed. SNL designed and built SPR II which incorporated a small central irradiation cavity. The cavity, of $2.54 \mathrm{~cm}$ usable diameter and $20.3 \mathrm{~cm}$ length and located on the vertical centerline of the reactor core, permitted the irradiation of test samples to fluences of up to $8 \mathrm{e} 14 \mathrm{n} / \mathrm{cm}^{2}$ (1-Mev-eq-Si) during pulses with a full width at half maximum (FWHM) as small as $40 \mu \mathrm{s}$. Operations 

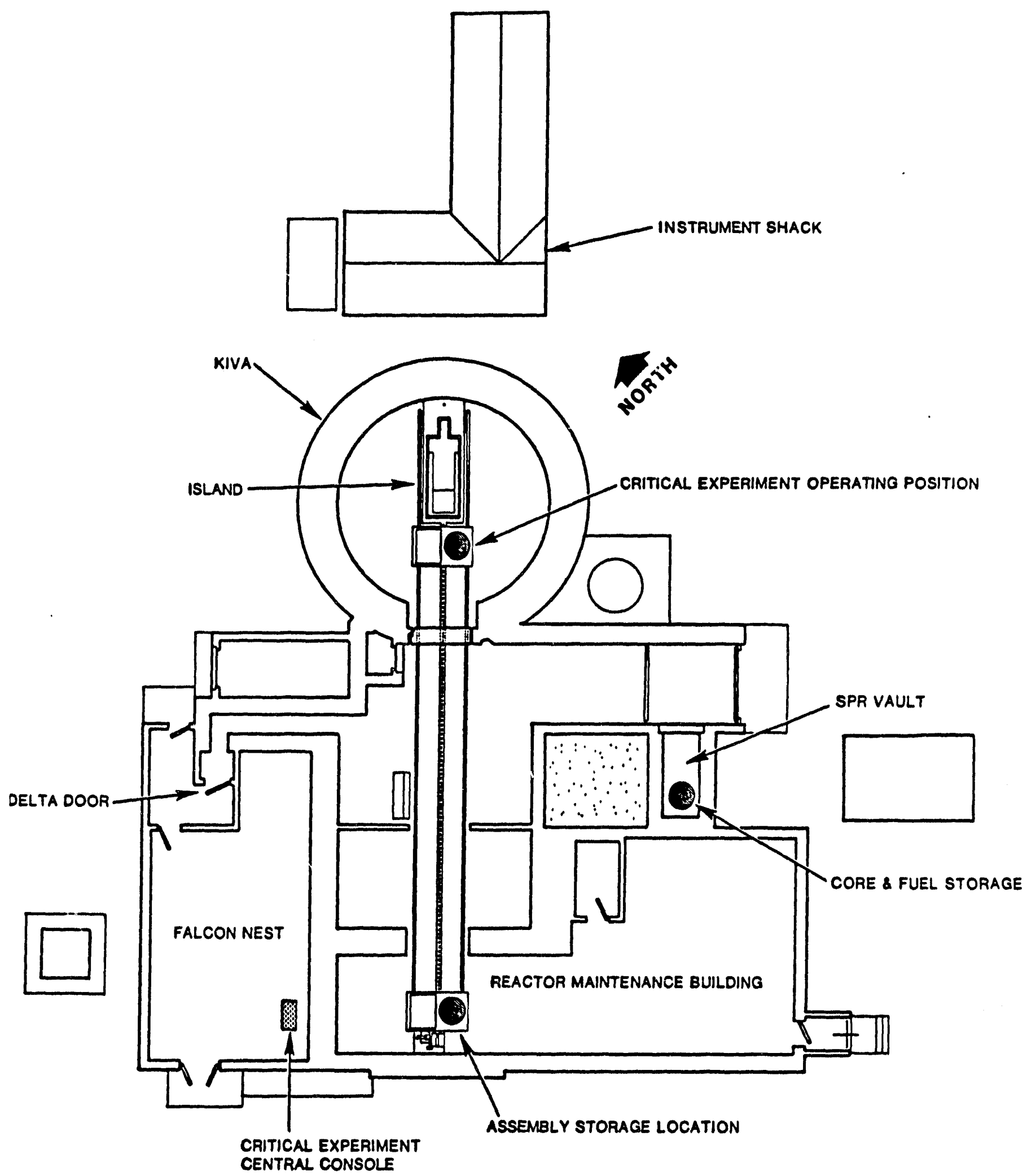

FIGURE 1. SPR Facility Layout 
- The irradiation of live surrogates to predict the effects of mixed radiation environments on military personnel;

- The first U.S. tests to investigate the existence of lasers induced through fission pumping;

- Pulse experiments on uranium and plutonium samples to provide data on the temporal response to fission induced shock in these metals.

\section{SPR III}

As the SPR customer base broadened with SPR II, more uses of fast burst reactors were proposed. To accommodate the new requirements, SNL designed and built the SPR III in 1975. The most significant increase in experiment capability was the expansion in size of the central irradiation cavity over that of SPR II to a usable diameter of $16.8 \mathrm{~cm}$ and a length of $36.8 \mathrm{~cm}$, a 79 fold improvement in volume. Once again, the increased capability permitted experimenters to increase the scope of their studies. New experiments containing explosives, fiber optics, accelerometers, radars, communications equipment, and liquids were conducted. Space programs benefiting from the increased capability included, but were not limited to, Galileo, Casini, MILSTAR, and Global Positioning Satellite. The larger cavity allowed the use of thermoelectric heaters and coolers to be used to create prototypic thermal environments on precision voltage and hard magnetic references. The cavity volume increase led directly to a quantum improvement in the study of fission activated lasers, because it was possible to tailor the pump rate to the gas mix and thereby optimize beam output. The steady state and pulse attributes of SPR III were in demand; the cavity increase permitted entire boxes of $10 \mathrm{~cm}$ diameter silicon wafers to be exposed during steady state operations to an inherently high fast neutron-to-gamma ratio to eliminate latch up. SPR III was used for such specialty applications as improving the precision of fast circuits used for timing inertial confinement fusion experiments and optimizing the output of diamond detectors of transient gamma pulses. In 1983, major modifications to the reactor and facility were completed to minimize the radiation exposure to experimenters and staff who needed to enter the reactor room to work on experiments or the reactor. In addition, a remotely controlled experiment cart was built so that experiments could be performed without the need for experimenters or staff to enter the reactor room. To date, nearly 10,000 operations have been conducted on SPR III, which is still in operation.

\section{SPR IIIM}

A modernized version of SPR III, SPR IIIM (Figure 2), will become operational at the facility in the summer of 1994. The usable diameter of the central irradiation cavity will be $19 \mathrm{~cm}$, so that, with a length $36.8 \mathrm{~cm}$, the volume will increase by $27 \%$ over SPR III. Single pulse fluences will be up to $6 e 14 \mathrm{n} / \mathrm{cm}^{2}$ (1-Mev-eq-Si) in the cavity. The free field reactor FWHM will be cumparable to SPR III at $90 \mu \mathrm{s}$ for a maximum pulse. The reactor will feature a forced cold nitrogen gas cooling system to permit higher steady state power levels (and shorter run times to achieve higher fluences). At $20 \mathrm{~kW}$, for example, it is expected that SPR IIIM will generate fluxes in the vicinity of $1 \mathrm{e} 12 \mathrm{n} / \mathrm{cm}^{2}-\mathrm{s}(1-\mathrm{Mev}-\mathrm{eq}-\mathrm{Si})$. In addition to the larger cavity diameter, the new reactor will feature an improved fuel design, larger diameter control element support shafts with precision bearings for improved stability, and a spherical bearing for the safety block drive for improved safety block seating. These features will provide improved pulse yield reproducibility and predictability and will reduce maintenance. The increase in cavity size and the increase in reactivity worth of the control elements will combine to permit experiments with more negative worth. As examples, moderating materials which convert neutrons to gamma rays can be used in the cavity to enhance the gamma environment at the expense of neutron fluence, and, fissile materials can be used in conjunction with moderators to function as pulse stretchers. As is the case with SPR III, the neutron energy spectra for both free field and customized radiation environments will be mapped in detail and well documented.

\section{THE CRITICAL ASSEMBLY}

As a parallel development with SPR operations since 1988, a critical assembly was designed, built, and operated at the SPR Facility. The initial purpose of the assembly was to perform measurements of key reactor parameters in order to benchmark the computer calculations and thereby refine the designs for a planned space propulsion 


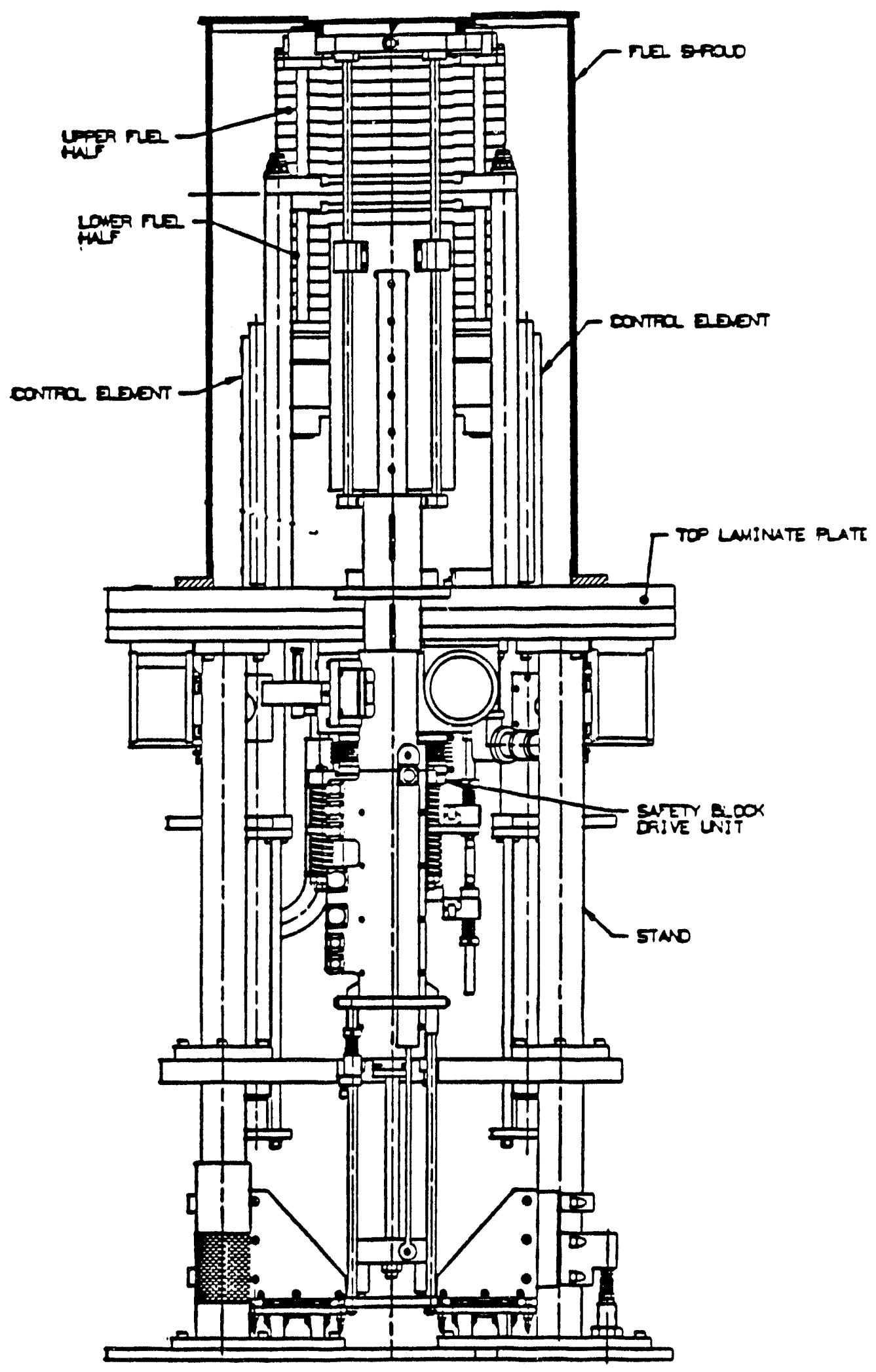

FIGURE 2. SPR IIIM ; total beight $1.9 \mathrm{~m}$ 
reactor. The fuel for the assembiy is a blend of small particles made from uranium carbide, zircaloy, and carbon. All the essential hardware for the assembly (core and dump tanks, control and safety rods, water pumps, and neutron detectors) was made portable so that assembly operation could take place serially with SPR operations. The small size of the fuel assembly $(67 \mathrm{~cm}$ diameter and $53 \mathrm{~cm}$ height), coupled with the nonhomogeneity of the fuel, made it difficult to analytically predict power profiles. Direct measurements of radial and axial profiles were performed to provide analysts with benchmark data. An interesting early result of the project was the discovery of an unanticipated positive temperature coefficient of reactivity. This finding alone underscores the utility and economy of conducting appropriately scaled mock-up testing of nuclear assemblies.

\section{NEW CRITICAL ASSEMBLIES}

As a outgrowth of success with the critical assembly, the SPR Facility is being considered for two additional criticals. The first is intended to address calculational uncertainties in the loading of power plant spent fuel into transportation containers and for geologic storage. A series of criticals is needed to validate a number of fuel types and loading geometries before the Nuclear Regulatory Commission authorizes movement of fuel in more compact, yet criticality safe, configurations.

Further into the future, the DOE intends to make a significant capital committment to the construction of the Advanced Neutron Source, a high very high flux, compact reactor for basic nuclear physics research. Because of the inhomogeneity of its core design, the ANS Program is evaluating sices to perform a confirmatory set of experiments on the nuclear design. The SPR Facility is one of the prope sed candidate sites for this activity.

\section{CHALLENGES}

Space nuclear power programs have emphasized the development of radiation hardened s,emiconductors for use in safety and control circuits of space-based systems. To that end, silicon carbide devices are currently of interest for space-based reactor control and safety because of their radiation tolerence at high fluences. A missing feature of these investigations is a corresponding concern for the performance of such devices during reactor transients. To properly qualify a protection system for space application requires testing in environments closely resembling excursions from which the plant should be capable of recovering. This implies taking preirradiated circuits (aging) through fast neutron pulses at prototypic temperatures. SPR III/IIIM could play an imporatant role as a platform for the required testing.

In addition, because the displacement damage from protons has been shown to be proportional to nonionizing energy deposition in electronic materials, the SPR Facility could be effectively and economically used to simulate damage from protons in space.

\section{CONCLUSIONS}

The SPR Facility has actively participated in resolving radiation hardness questions for over thirty years. A necessary consequence to that participation has been a number of contributions to the improvement in capability of fast burst reactors. But as national priorities have changed, so has the focus of the facility, addressing new initiatives in space nuclear power, transportation, and fundamental reactor research, in addition to the testing of electronics for weapons systems. 


\section{Acknowledgements}

Funding for these projects is being provided by the U.S. Department of Energy under contract number DE-AC0476DP00789. Special thanks are extended to the staff and management of SNL/Technical Area V.

\section{Bibliography}

Bonzon, L.L., B.F. Estes, and J.S. Philbin (1981), Sandia Pulsed Reactor II (SPR II): Safety Analysis Report, SAND81-1264, Albuquerque: Sandia National Laboratories.

Bonzon, L.L., B.F. Estes, J.S. Philbin, and J. Reuscher (1978), Sandia Pulsed Reactor III (SPR III): Safety Analysis Report, SLA74-0349, Albuquerque: Sandia National Laboratories.

Hoovler, G.S. (1992), Radial Fission Power Distribution in the Critical Experiment (CX) Fuel Stalks, BSW Report ASE-58-3001806-00, Babcock and Wilcox.

Kelly, J.G., P.T. Griffin, W.C. Fan (1993) "Benchmarking the Sandia Pulsed Reactor III Cavity Neutron Spectrum for Electronic Parts Calibration ans Testing," in IEEE Transactions on Nuclear Science, 40(6). 

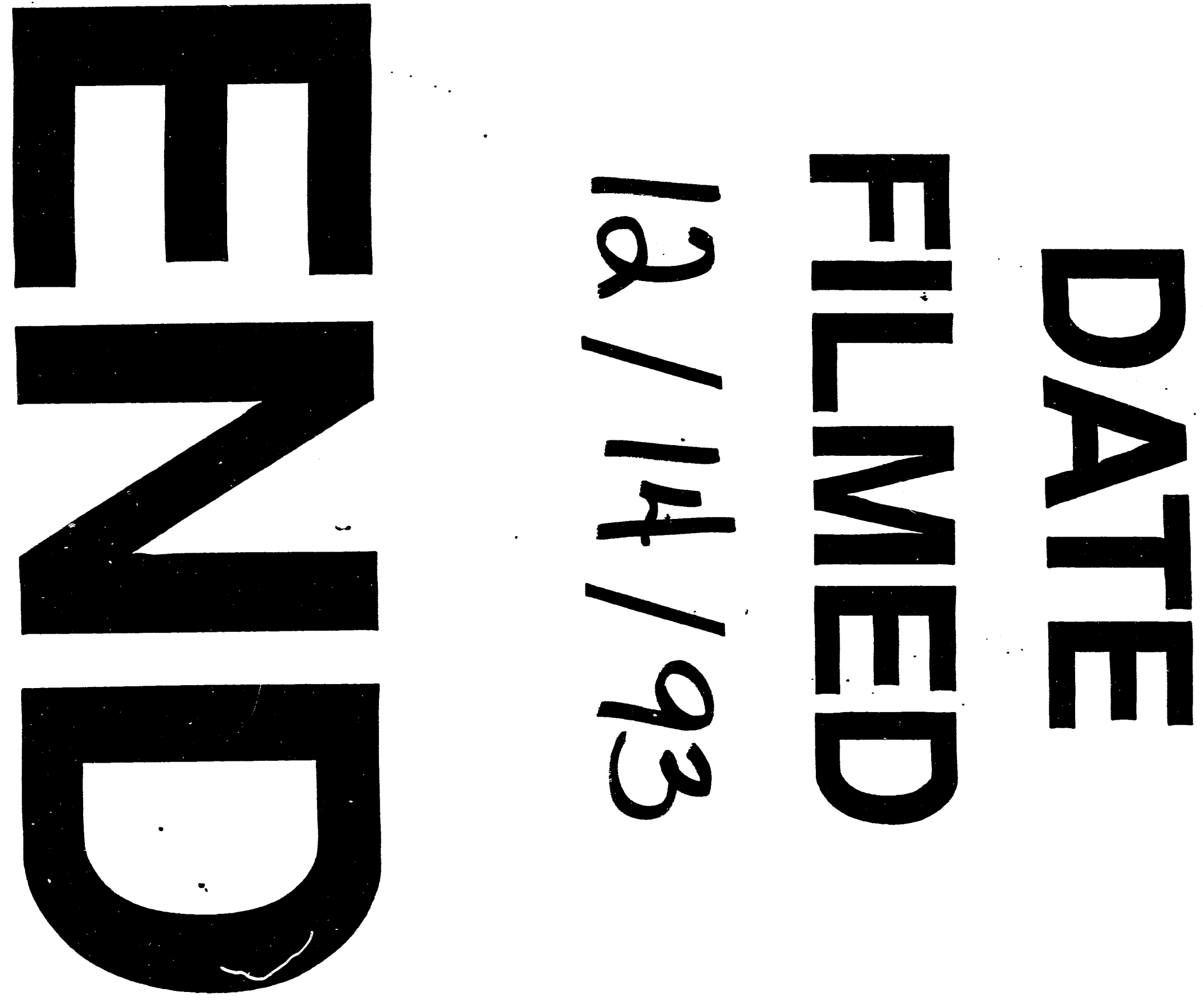


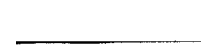

